\title{
Metal-Semiconductor-Metal $\varepsilon-\mathrm{Ga}_{2} \mathrm{O}_{3}$ Solar-Blind Photodetectors with
}

\section{a Record High Responsivity Rejection Ratio and Their Gain}

\section{Mechanism}

Yuan Qin,\# Liheng Li,\# Xiaolong Zhao,\# Gary S. Tompa, Hang Dong, Guangzhong Jian, Qiming He, Pengju Tan, Xiaohu Hou, Zhongfang Zhang, Shunjie Yu, Haiding Sun,* Guangwei Xu, Xiangshui Miao,

Kanhao Xue,* Shibing Long,* and Ming Liu

Y. Qin, H. Dong, G. Jian, Q. He, Prof. M. Liu

Key Laboratory of Microelectronics Devices \& Integration Technology

Institute of Microelectronics of Chinese Academy of Sciences

Beijing 100029, China

Dr. X. Zhao, P. Tan, X. Hou, Z. Zhang, S. Yu, Prof. H. Sun (haiding@ustc.edu.cn), Dr. G. Xu, Prof. S. Long (shibinglong@ustc.edu.cn)

School of Microelectronics

University of Science and Technology of China

Hefei, Anhui 230026, China

Y. Qin, H. Dong, G. Jian, Prof. M. Liu

School of Electronic, Electrical and Communication Engineering

University of Chinese Academy of Sciences

Beijing 100049, China

Prof. S. Long, Prof. M. Liu

Jiangsu National Synergetic Innovation Center for Advanced Materials (SICAM)

Nanjing, Jiangsu 210023, China

G. S. Tompa

Structured Materials Industries Inc.

Piscataway, New Jersey 08854, United States.

L. Li, Prof. X. Miao, Prof. K. Xue (xkh@hust.edu.cn)

Wuhan National Laboratory for Optoelectronics, School of Optical and Electronic Information

Huazhong University of Science and Technology

Wuhan 430074, China 


\section{Supporting Information}

\section{Content list:}

Figure S1: Transmittance spectrum of the $\varepsilon-\mathrm{Ga}_{2} \mathrm{O}_{3}$ film.

Figure S2: Arrhenius plot of the conductance of the MSM $\varepsilon-\mathrm{Ga}_{2} \mathrm{O}_{3}$ SBPD in the dark.

Figure S3: Semi-log response spectrum of the $\mathrm{MSM} \varepsilon-\mathrm{Ga}_{2} \mathrm{O}_{3} \mathrm{SBPD}$ at different bias.

Figure S4: Peak responsivity of the spectral response spectrum as a function of square root of the bias voltage.

Figure S5: Temperature-dependent current-voltage characteristics photoexcited by 254 nm light.

Figure S6: Temperature-dependent photocurrent and responsivity characteristics with temperature varying from 300 to $500 \mathrm{~K}$.

Figure S7: Structure diagrams of the $\varepsilon-\mathrm{Ga}_{2} \mathrm{O}_{3}$ with $\mathrm{H}$ or $\mathrm{Cl}$ dopants.

Figure S8: Cathodoluminescence spectrum of the $\varepsilon-\mathrm{Ga}_{2} \mathrm{O}_{3}$ film.

Figure S9: Schematic band diagram of the $\varepsilon-\mathrm{Ga}_{2} \mathrm{O}_{3}$. 


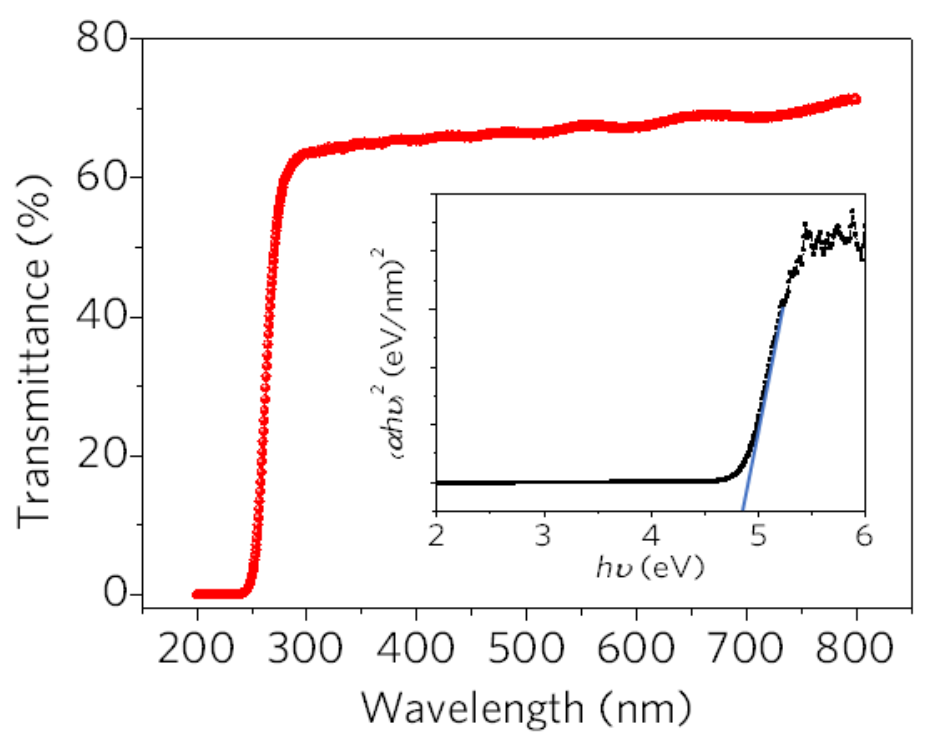

Figure S1. The transmittance spectrum of the $\varepsilon-\mathrm{Ga}_{2} \mathrm{O}_{3}$ film. Inset shows the Tauc plot $(\alpha h v)^{2}$ vs $h v$. The transmittance drops abruptly when the wavelength is below $280 \mathrm{~nm}$, indicating a strong absorption beyond this wavelength, confirming the solar-blind characteristics of the film. The band gap of the $\varepsilon-\mathrm{Ga}_{2} \mathrm{O}_{3}$ film was extracted to be about $4.9 \mathrm{eV}$.

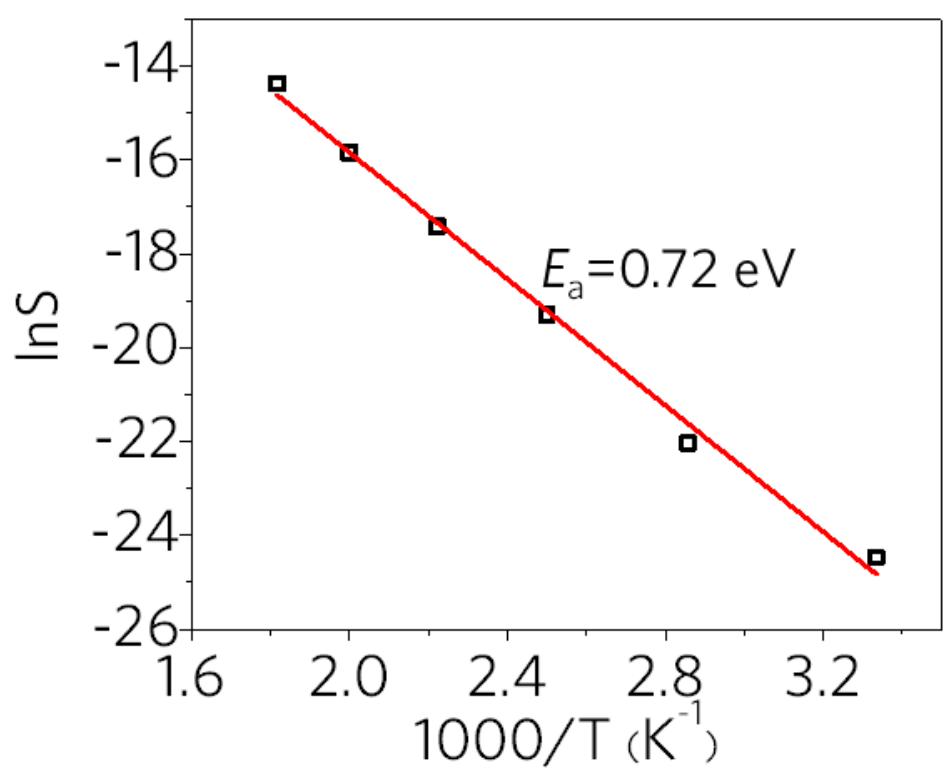

Figure S2. Arrhenius plot of the conductance of the MSM $\varepsilon-\mathrm{Ga}_{2} \mathrm{O}_{3}$ SBPD in the dark. 
in the temperature range from $300 \mathrm{~K}$ to $500 \mathrm{~K}$. S denotes the ratio of current and voltage of the MSM $\varepsilon-\mathrm{Ga}_{2} \mathrm{O}_{3}$ SBPDs in the dark. The activation energy of $0.72 \mathrm{eV}$ can be extracted from the slope of $\ln S v s .1000 / \mathrm{T}$, which is related to thermal ionization of deep defect state located at about $0.7 \mathrm{eV}$ below the conduction band.

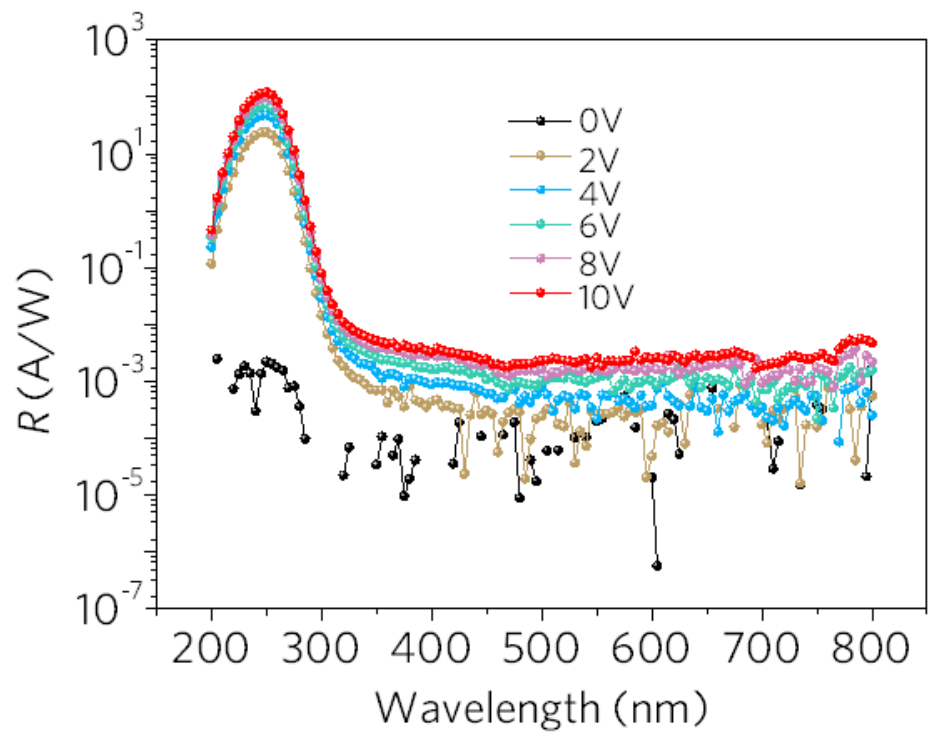

Figure S3. Response spectrum of the MSM $\varepsilon-\mathrm{Ga}_{2} \mathrm{O}_{3}$ SBPD in a semi-log scale at different bias voltages at room temperature. 


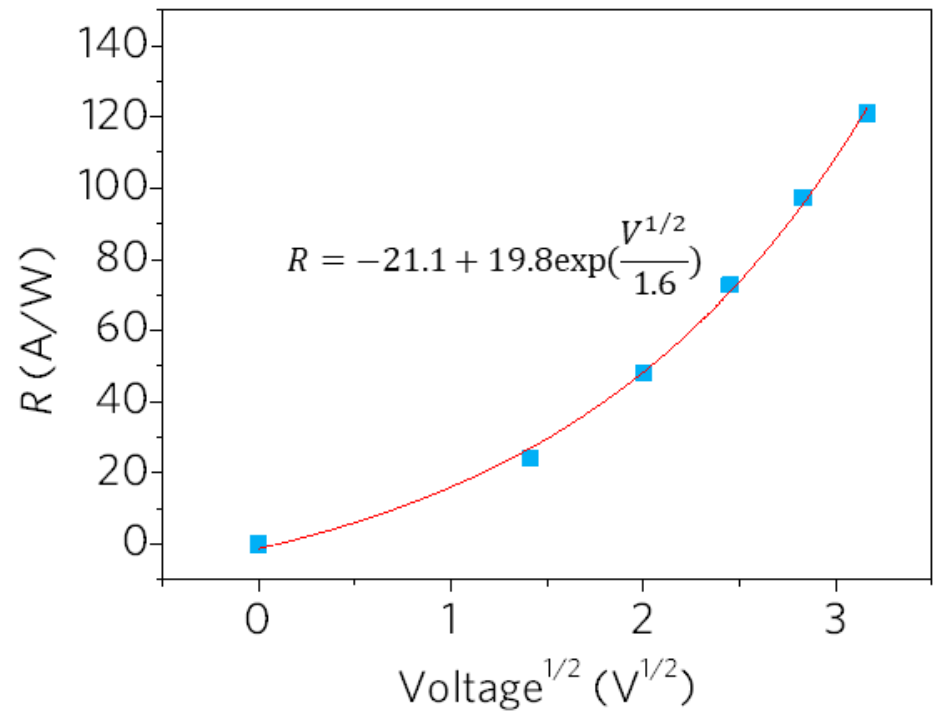

Figure S4. Peak responsivity of the spectral response spectrum as a function of square root of the bias voltage.

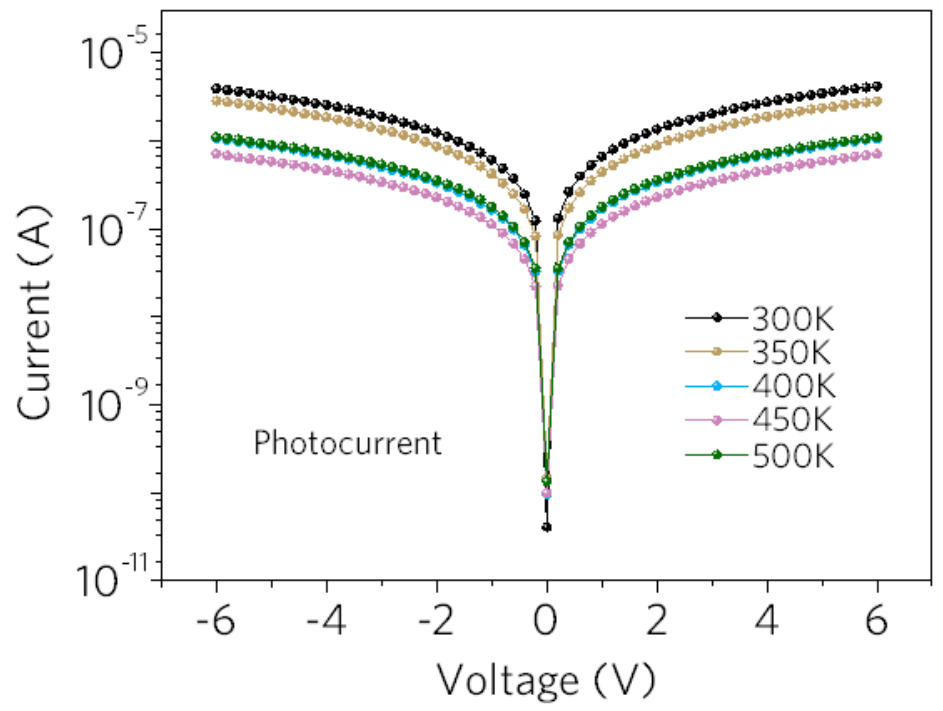

Figure S5. Temperature-dependent $I-V$ characteristics of the MSM $\varepsilon-\mathrm{Ga}_{2} \mathrm{O}_{3} \mathrm{SBPD}$ on a semi-log under $254 \mathrm{~nm}$ light illumination. 


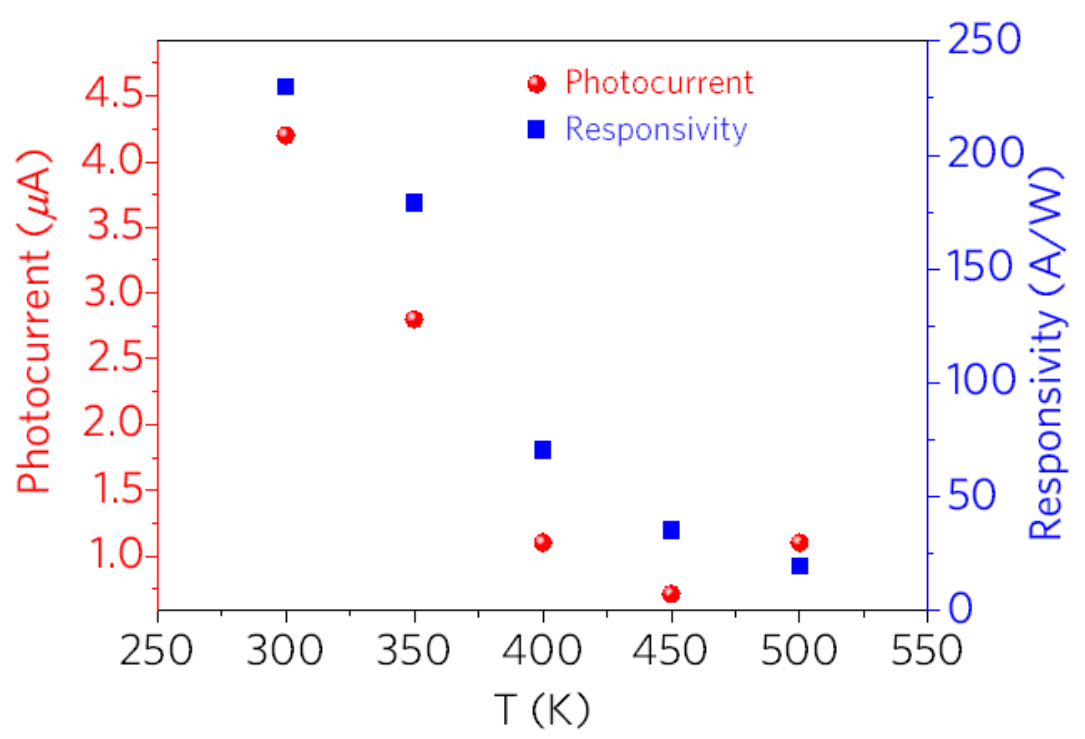

Figure S6. Photocurrent and responsivity as a function of temperature in the range from 300 to $500 \mathrm{~K}$ with a step of $50 \mathrm{~K}$. The photocurrent and responsivity are observed to decrease at higher temperature, indicating the current gain is suppressed.
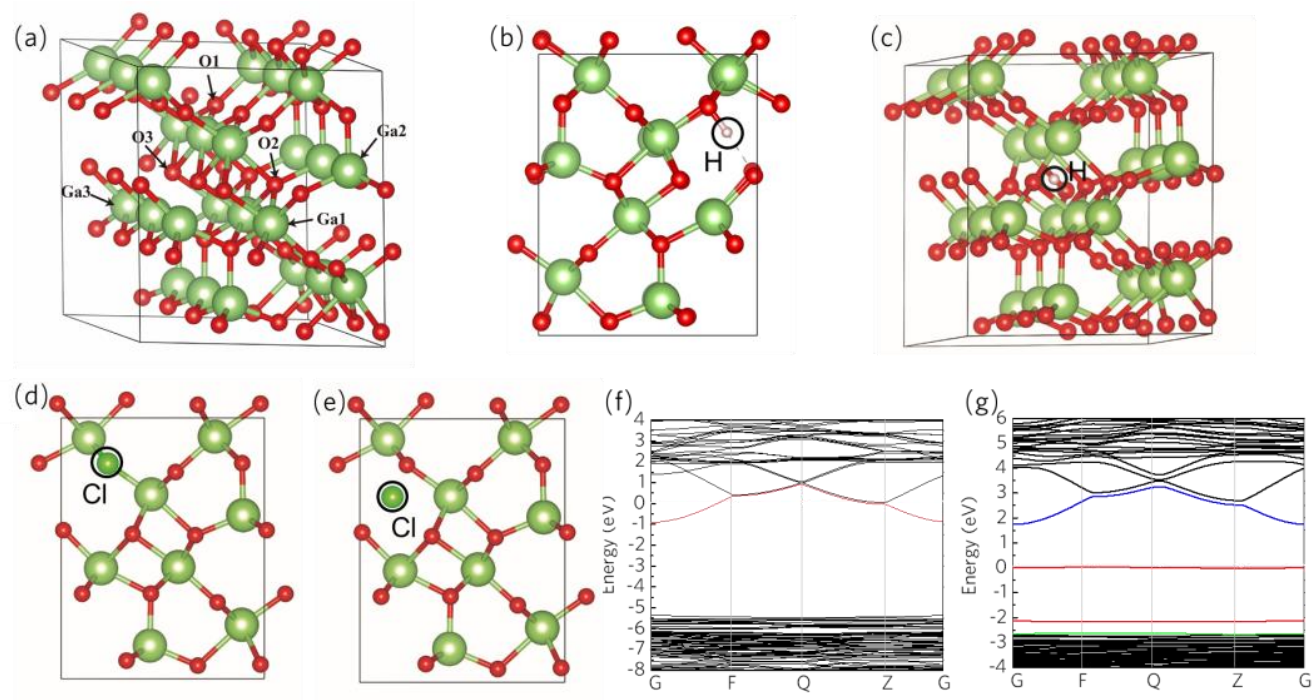

Figure S7. (a) Supercell structure of $\varepsilon-\mathrm{Ga}_{2} \mathrm{O}_{3}$. Structure diagrams of $\mathrm{H}$ as (b) interstitial dopant and (c) substitutional dopant. Structure diagrams of $\mathrm{Cl}$ as (d) substitutional dopant and (e) interstitial dopant. Density of states of (f) H interstitial dopant and (g) 
Cl interstitial dopant.

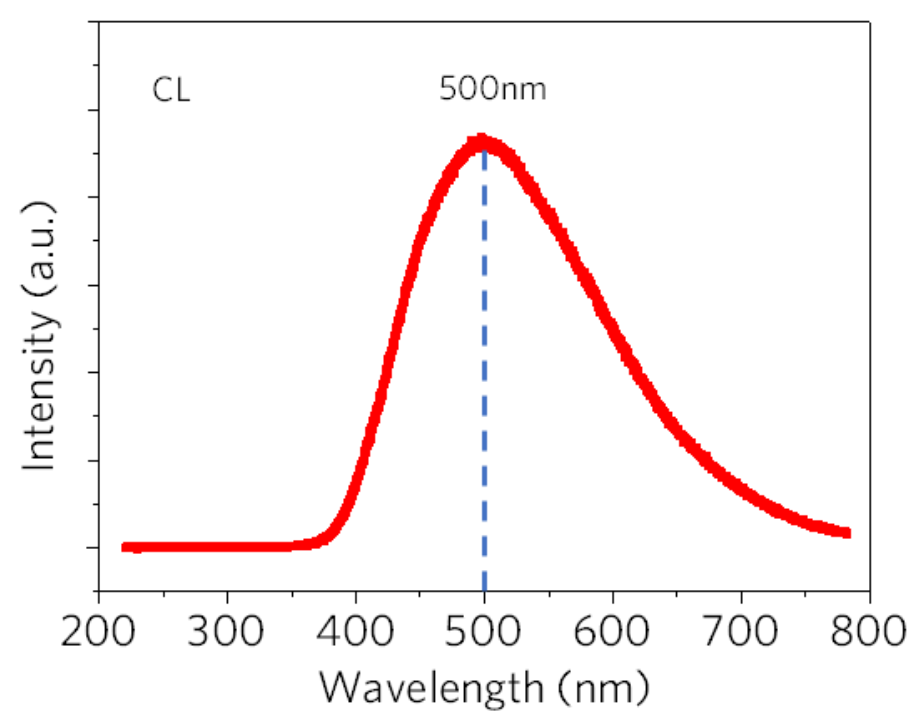

Figure S8. Cathodoluminescence spectrum of the $\varepsilon-\mathrm{Ga}_{2} \mathrm{O}_{3}$ film with a broad defect peak centered around $500 \mathrm{~nm}$.

The CL spectrum shows the presence of a broad defect-related peak centered around $500 \mathrm{~nm}$. This blue emission is due to donor-acceptor recombination at intrinsic vacancys. In this case, it occurs by a recombination of electrons at the donor to the hole at the acceptor via neutral defects, such as oxygen vacancy. 


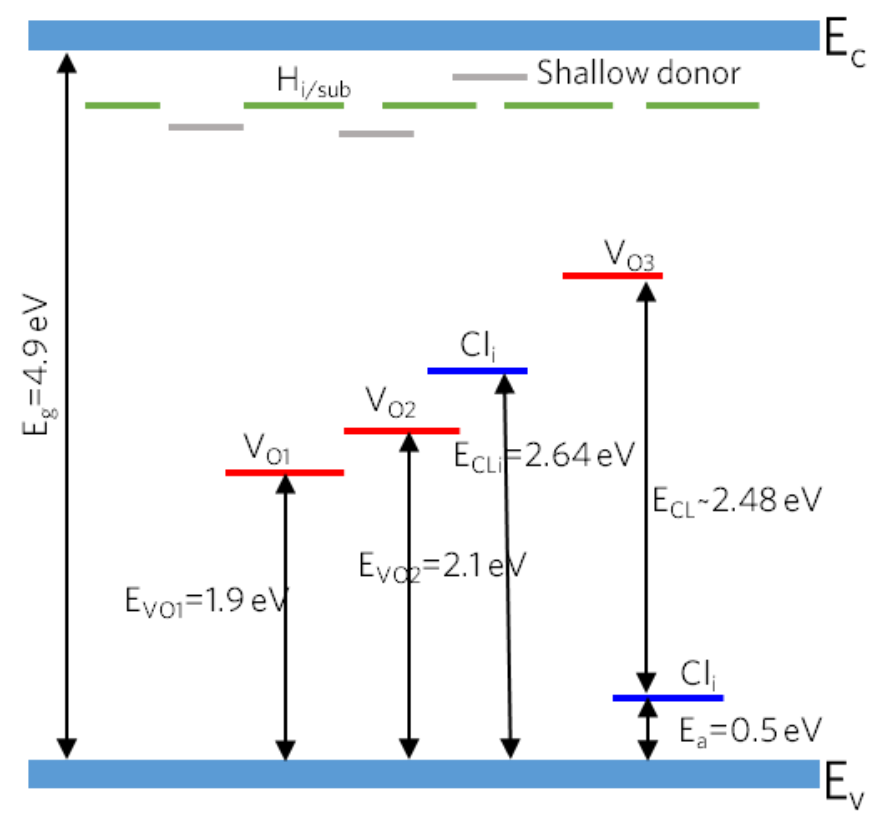

Figure S9. Schematic band diagram of the $\varepsilon-\mathrm{Ga}_{2} \mathrm{O}_{3}$.

According to the DFT calculation, oxygen vacancies are formed in the $\varepsilon-\mathrm{Ga}_{2} \mathrm{O}_{3}$ film for three inequivalent oxygen. $\mathrm{O} 1$ and $\mathrm{O} 3$ are 3-fold coordinated, while $\mathrm{O} 2$ is 4-fold coordinated. The introduced deep-level defect states of oxygen vacancy are positioned $2.10,1.91$ and $2.85 \mathrm{eV}$ above the maximum of valence band for $\mathrm{O} 1, \mathrm{O} 2$ and $\mathrm{O} 3$, respectively. The $\mathrm{Cl}$ interstitial dopant introduces two defect levels in the sub band gap, including a deep level defect state positioned at $2.64 \mathrm{eV}$ above the maximum of valence band and a shallow acceptor level. H can passivate deep level defect states and acts as shallow donor in $\mathrm{Ga}_{2} \mathrm{O}_{3}$. As is shown in Figure $\mathrm{S} 9$, a broad defect-related peak centered around $500 \mathrm{~nm}$ is observed in the CL spectrum. The blue emission is caused by recombination of electrons at the donor to the hole at the acceptor via neutral defects, such as oxygen vacancy. In this case, it can be explained by the recombination of electrons in the donor state and holes in the acceptor introduced by $\mathrm{Cl}$ interstitial dopant via neutral oxygen vacancy $(\mathrm{O} 3)$. The energy difference of between $\mathrm{V}_{\mathrm{O} 3}$ and $\mathrm{Cl}_{\mathrm{i}}$ is 
roughly consistent with energy of the luminescent peak in the CL spectrum. 